\title{
PREVALENCE OF OCULAR MANIFESTATIONS IN PATIENTS HOSPITALIZED WITH DENGUE FEVER
}

Narendra P. Datti¹, B.N. Raghavendra Prasad ${ }^{2}$, Bere Gowda ${ }^{3}$, Prashanth Krishnappa ${ }^{4}$, Mithila R ${ }^{5}$, Trupti Gowda6

\author{
HOW TO CITE THIS ARTICLE: \\ Narendra P. Datti, B.N. Raghavendra Prasad, Bere Gowda, Prashanth Krishnappa, Mithila R, Trupti Gowda. \\ "Prevalence of Ocular Manifestations in Patients Hospitalized with Dengue Fever". Journal of Evolution of \\ Medical and Dental Sciences 2014; Vol. 3, Issue 07, February 17; Page: 1704-1708, \\ DOI: $10.14260 /$ jemds/2014/2045
}

ABSTRACT: AIM: To study the prevalence of ocular manifestations in seropositive dengue patients. METHODS: Cross sectional observational study was done at R.L. JALAPPA hospital. IgM positive dengue cases were selected over 6 week period of a dengue epidemic and ocular examination was carried out. RESULTS: A total of 142 eyes of 71 patients were examined in which $40(56.3 \%)$ were males and 31(43.6\%) females. Age of patients ranged from 3 to 60 years (mean-24 years). 40 eyes $(28.5 \%)$ of 21 patients had ocular manifestations. 21 eyes $(52.5 \%)$ had anterior segment findings in form of petechial and subconjunctival hemorrhages. 19 eyes (47.5\%) had posterior segment findings like cotton wool spots, dot and blot hemorrhages, macular edema, hyperemic disc and optic neuritis. 17 patients with platelet count of $<1,00,000 / \mu l$ had ocular manifestations. $\mathrm{P}$ value was not significant because of limited subjects. CONCLUSION: The prevalence of ocular manifestations in patients of dengue in our setup was $28.1 \%$. Ocular manifestations were more common in patients with decreased platelet count and it's advisable for a detailed ocular examination in patients with thrombocytopenia to avoid any residual impairment of vision.

KEYWORDS: dengue, macular edema, ocular manifestation, prevalence.

INTRODUCTION: Dengue is self-limiting common arthropod-borne viral disease which is transmitted to humans by mosquitoes. It is endemic in tropics and warm temperate regions of the world. It is caused by 4 antigenically similar but immunologically distant serotypes of dengue virus of genus flavi virus transmitted by Aedes Aegypti mosquito. Highest incidence occurs in South Asia and American tropics. ${ }^{1}$

Dengue hemorrhagic fever is a more severe form of disease characterized by multisystem hemorrhagic manifestation, increased vascular permeability and plasma leakage. In dengue fever, patient presents with abrupt onset fever after a 2-7 day incubation period with body temperature reaching 41 degree Celsius. Symptoms include severe malaise, arthralgia, headache, retro orbital pain and lumbosacral pain ${ }^{1-3}$. Patient presents with morbiliform rashes on trunk, spreading centripetally to face, trunk and limbs. Palms and soles are spared. ${ }^{3}$ Patients experience respiratory symptoms like sore throat, nausea and also have relative bradycardia and lymphadenopathy. Blood investigations show neutropenia, leucopenia and thrombocytopenia. Hemorrhagic complications are usually mild and limited to gum bleeding, epistaxis, hematuria or menorrhagia ${ }^{4}$. The reported ocular changes are petechial and subconjunctival hemorrhage, retinal edema, blot hemorrhages, vasculitis, exudative retinal detachment, cotton wool spots and anterior uveitis. ${ }^{2}$

The expansion of this flavi virus is linked to resurgence of the mosquito vector Aedes Aegypti, uncontrolled urbanization and overcrowding without appropriate water management and global 
spread of dengue via travel and trade. Dengue is emerging as a most important arthropod borne viral disease after malaria.

MATERIALS AND METHODS: This was a cross sectional observational study in which consecutive patients hospitalized with dengue fever over a 6- week period between May-July 2012 at R.L.J Hospital, Kolar were enrolled. Diagnosis was made by physician and pediatrician on basis of characteristics clinical signs and symptoms. Diagnosis was confirmed by using was done by using dengue IgM capture ELISA test.

After Informed consent, a detailed clinical history including systemic and visual complaints was taken. Patients with history of systemic disorders and ophthalmic disorders including diabetes mellitus, hypertension, and anemia were excluded. Patients underwent visual acuity, anterior segment examination by slit lamp and posterior segment examination by dilated fundoscopy.

Patients with ocular findings were called for weekly follow up upto 6 weeks in ophthalmology clinic after they were discharged from the hospital.

RESULTS: A total of 142 eyes of 71 patients were examined for any ocular manifestation of dengue. Out of 71 patients $40(56.3 \%)$ were males and 31(43.6\%) were females. Age of patients ranged from 3 years of age to 60 years (mean age of 24 years).

$20(28.1 \%)$ patients presented with headache which was the most common symptom. $7(9.85 \%)$ patients presented with retro orbital pain. $2(2.8 \%)$ patients complained of blurring of vision.

Out of 142 eyes, $40(28.1 \%)$ eyes had positive ocular findings. 102 (71.8\%) had no positive ocular findings. Our commonest anterior segment finding was petechial hemorrhage in 17 (42.5\%) eyes and $4(10 \%)$ eyes had subconjuctival hemorrhage.

19(47.5\%) eyes had posterior segment findings.6 (15\%) eyes had macular edema. 3(7.5\%) eyes had intraretinal bleed in form of dot and blot haemorrhages.2 (5\%) of eyes had cotton wool spots in unilateral eyes.

All patients laboratory parameters were examined in comparison with ocular findings in which it was found that $17(42.5 \%)$ patients had thrombocytopenia $(<1 \mathrm{lakh} \mu / \mathrm{l})$. Follow up of patients revealed resolution of subconjunctival hemorrhage in 3-4 weeks of time. There was resolution in posterior segment findings which resolved in 2-6 weeks of time without specific treatment.

DISCUSSION: The incidence, geographic distribution, and clinical severity of epidemic and endemic have increased in past few decades ${ }^{5}$.Dengue hemorrhagic fever is defined by WHO as dengue fever associated with thrombocytopenia ( $<1$ lakh cells/lt) and hemoconcentration (hematocrit elevated $>20 \%$ above baseline)6. Dengue shock syndrome is a severe form of disease associated with hypotension, narrowing of pulse pressure $(<20 \mathrm{mmHg})$ and circulatory failure in $30 \%$ of cases. The mortality rate for untreated DHF can be as high as $10-15 \%$ in places where patients have no ready access to emergency supportive therapy with i.v. fluids and platelet infusion ${ }^{6}$ worldwide case of dengue illness exceeds 100 million/year. 25, 000 deaths are reported annually to WHO due to dengue hemorrhagic fever6,3. 
Ocular findings are usually seen 4-5 day after onset of fever. Direct viral infection of dendritic cells cause apoptosis and dysfunction. A transient aberrant immune response may occur leading to cytokine overproduction and CD4/CD8 inversion. Overproduction of IL-6 may trigger production of auto antibodies against platelets and endothelial cells.1,7 There is a postulation that the vasculitis may be done to immune complex deposition on the walls of the small ocular blood vessels. Dengue viremia incites antibody production which if overwhelming, causes deposition of viral antigen-antibody immune complexes resulting in inflammation of retinal vessels ${ }^{8}$.

Kapoor et al studies showed prevalence of ocular findings in $40.3 \%$ of patients ${ }^{2}$. In Kapoor et al studies $37.3 \%$ patients had anterior segment findings in form of petechial and subconjunctival hemorrhage. In our study, the prevalence was $28.1 \%$. In our study, majority of patients $42.5 \%$ had petechial hemorrhage and $7.5 \%$ of patients had subconjunctival hemorrhage. [Fig. 1, 2].

Lim et al studies reported intra retinal whitish lesions with localized retinal and retinal pigment epithelium disturbance, small dot hemorrhages, vascular sheathing around macula papillomacular bundle ${ }^{9}$ Haritoglou et al studies revealed intraretinal hemorrhages, retinal pigment epithelial lesions in fovea, cotton wool spots and maculopathy ${ }^{10}$.

Cruz-villegas studies also reported posterior bilateral choroidal effusion, ${ }^{11}$ in our studies, the posterior segment findings were in form of macular edema (15\%), dot and blot hemorrhages (7.5\%) and cotton wool spots (5\%).

Gomber et al reported that there was no significance association between thrombocytopenia and hemorrhagic manifestations signifying that there may be other factors. Kapoor et al reported had $90.7 \%$ of patients with ocular hemorrhage had marked thrombocytopenia. In our study, $42.5 \%$ of patients had significant thrombocytopenia (<1lakh cells $/ \mathrm{lt})^{12}$

According to Haritoglou et al most of the positive fundal findings resolve without specific treatment.10-12 In our study 19 patients has posterior segment findings who were reexamined resolved without any specific treatment in 2-6 weeks.

CONCLUSION: Visual Prognosis is generally good as disease is often self-limiting and resolves spontaneously with supportive treatment. ${ }^{13}$ The prevalence of ocular manifestations in patients with dengue in our setup was $28.5 \%$. Ocular manifestations were more common in patients with decreased platelet count. Such patients are predisposed to spontaneous ocular hemorrhage which is usually subconjunctival. If present in macula, it can lead to impairment of vision. Ophthalmologist should be aware of various manifestation of dengue. It is advisable for a detailed ocular examination in patients with thrombocytopenia to avoid any residual impairment of vision.

\section{REFERENCES:}

1. Teoh SCB, Chan DPL, Nah GKM, Rajagopalan R, Laude A, Ang BSP, Barkham T, et al. A re-look at ocular complications in dengue fever and dengue haemorrhagic fever. Dengue bulletin 2006; 30:184-90.

2. Kapoor H K, Bhai S, John M, Xavier j. Ocular manifestations of dengue fever in an East Indian epidemic. Can J Ophthalmol 2006; 41:741-46.

3. Chan OPL, Teoh SCB, Tan CSH, Nah GKM, Rajagopalan R Prabhakar, Gupta MK, Chee CKL et al. Ophthalmic Complications of Dengue. Emerging infectious diseases 2006; 12:285-89. 
4. Chlebicki PM, Ang B, Barkham T, Laude A. Retinal haemorrhages in 4 patients with dengue fever. Emerging infectious diseases 2005; 11:770-2.

5. Gibbons RV, Vaughn DW. Dengue: an escalating problem. BMJ 2002; 324:1563-6.

6. World health organization. Dengue haemorrhagic fever: Diagnosis, treatment and control.2nd edn. Geneva, Switzerland: WHO; 1997.

7. Wilner ZH, Moisseiev J, Bin H, Rubinovitch B.A returned traveller with dengue and visual impairement. IMAJ 2005; 7:200-01.

8. Chang JPE, Cheng CL, Asok K, Fong KY, Chee SP, Tan CK. Visual disturbances in dengue fever: an answer at last? Singapore Med J 2007; 48:71-3.

9. Lim WK, Mathur R, Koh A, Yeoh R, Chee SP. Ocular Manifestations of Dengue Fever. Opthalmology 2004; 111:2057-64.

10. Harioglou C, Dotse SD, Rudolph G, Stephan CM, Thurau SR, Klaub V. A tourist with dengue fever and visual loss. Lancet 2002; 360:10. 70.

11. Cruz-Villegas V, Berrocal AM, Davis JL. Bilateral choroidal effusions associated with dengue fever. Retina 2003; 23:576-8.

12. Gomber S, Ramachndran VG, Kumar S, et al. Hematological observations and diagnostic markers in dengue haemorrhagic fever- a reappraisal. Indian pediatr 2001; 38:477-81.

13. Simmons CP, Farrar JJ, Chau NV, Wills B. Dengue. N Engl J Med 2012; 366:1423-32.

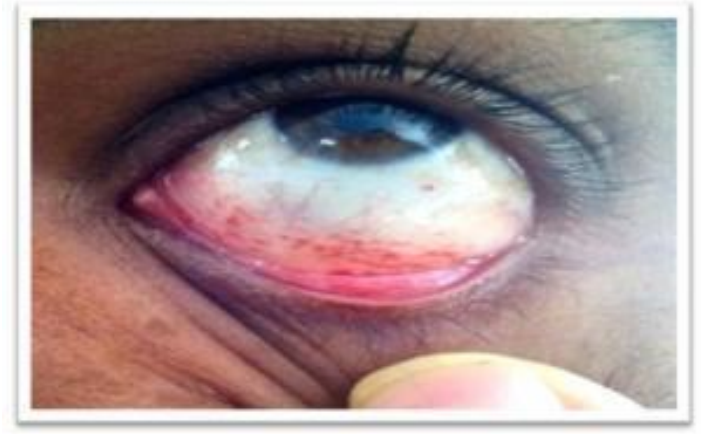

Fig. 1

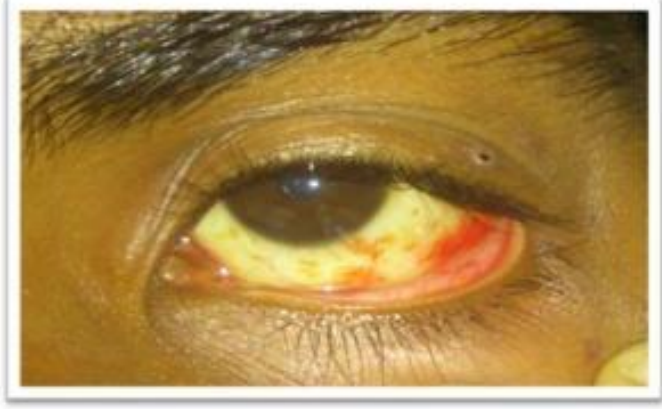

Fig. 2

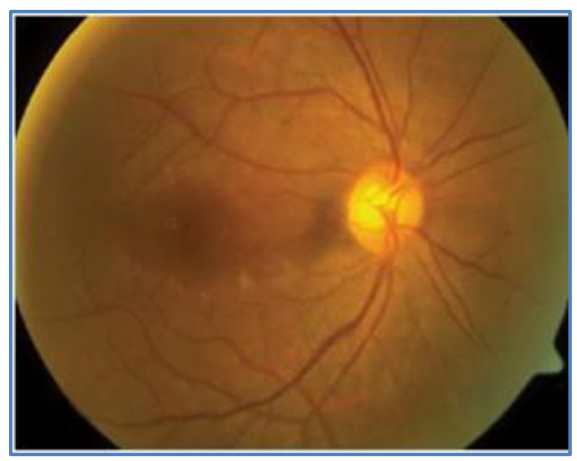

Fig. 3 


\section{ORIGINAL ARTICLE}

\section{AUTHORS:}

1. Narendra P. Datti

2. B.N. Raghavendra Prasad

3. Bere Gowda

4. Prashanth Krishnappa

5. Mithila R.

6. Trupti Gowda

\section{PARTICULARS OF CONTRIBUTORS:}

1. Professor and HOD, Department of Ophthalmology, Sri Devraj Urs Medical College.

2. Professor and HOD, Department of Medicine, Sri Devraj Urs Medical College.

3. Professor and HOD, Department of Pediatrics, Sri Devraj Urs Medical College.

4. Associate Professor, Department of Ophthalmology, Sri Devraj Urs Medical College.
5. Post Graduate Student, Department of Ophthalmology, Sri Devraj Urs Medical College.

6. Post Graduate Student, Department of Microbiology, Sri Devraj Urs Medical College.

\section{NAME ADDRESS EMAIL ID OF THE CORRESPONDING AUTHOR:}

Dr. Mithila R,

206, PG Womens Hostel,

Sri Devraj Urs Medical College,

Tamaka, Kolar - 563101.

E-mail: mithila.singh@yahoo.com

Date of Submission: 05/01/2014.

Date of Peer Review: 07/01/2014.

Date of Acceptance: 13/01/2014.

Date of Publishing: 13/02/2014. 